\title{
A Monte Carlo study of spectroscopy in nanoconfined solvents
}

\author{
Ward H. Thompson \\ Department of Chemistry, University of Kansas, Lawrence, Kansas 66045
}

(Received 2 May 2002; accepted 16 July 2002)

\begin{abstract}
The absorption and fluorescence spectra of a model diatomic molecule with a charge-transfer electronic transition are simulated. The effect of confining the solvent in which the diatomic molecule is dissolved is examined by comparing results for solutions contained within hydrophobic spherical cavities of varying size (radii of 10-20 ̊). The effect of solvent polarity is also considered by comparing results of simulations with $\mathrm{CH}_{3} \mathrm{I}$ and $\mathrm{CH}_{3} \mathrm{CN}$ solvents. The spectra, solute radial and angular distribution functions, and free energy surfaces in the solvent and radial solute position coordinates are presented and discussed. It is found that the solute position in the cavity critically affects the absorption and fluorescence spectra and their dependence on cavity size. The implications of these results for time-dependent fluorescence measurements are discussed. (C) 2002 American Institute of Physics. [DOI: 10.1063/1.1505436]
\end{abstract}

\section{INTRODUCTION}

It is now possible to synthesize nanometer-sized cavities in sol-gels, ${ }^{1}$ zeolites, organic, and inorganic supramolecular assemblies, ${ }^{2}$ reverse micelles, ${ }^{3}$ vesicles, and even proteins. ${ }^{4}$ This gives strong impetus to improving our understanding of chemical reactions and spectroscopy in nanoconfined solvents. For example, these cavities might serve as vessels in which chemical reactions take place in the small pool of solvent allowed in the restricted space. It is not difficult to imagine that the dynamics of such reactions can be significantly affected by the characteristics of the cavity including the size, shape, flexibility, and interactions with the solvent and/or reactants. Ideally, one wishes to control the chemistry not only by manipulating what species are allowed in the cavity but also by designing the cavity properties. However, to accomplish this a better understanding of the effect of nanoscale confinement and the cavity characteristics on reactivity and spectroscopy is needed.

Chemical processes that are strongly coupled to the solvent, typically those involving charge transfer such as electron and proton transfer reactions, ${ }^{5,6}$ should be most affected by confinement of the solvent. Thus they should exhibit some of the most interesting phenomena and provide the greatest opportunity for manipulating the chemistry. For these processes the limited number of solvent molecules, geometric constraints of a nanocavity, and solvent cavity interactions may have dramatic effects on both the energetics and dynamics. This is one of the motivations for the study of charge-transfer spectra presented in this paper.

The structure and dynamics of solvents in nanocavities of various types have attracted increasing attention in recent years. ${ }^{3,7-38}$ There have been numerous experimental studies of confined neat liquids ${ }^{7-9}$ and of solutes in confined solvents. ${ }^{9-27}$ Of particular interest for the present work are several investigations of solvation dynamics in a wide variety of nanocavity systems that have probed the timeindependent and time-dependent spectroscopy ${ }^{9-27}$ of chromophores dissolved in the confined solvents.
There have been relatively few studies that compare the solvation dynamics in a range of confinement frameworks. One example is the work of Bhattacharyya and co-workers who have measured the time-dependent Stokes shift (with $\sim 80$ ps resolution) of the same dye molecule, Coumarin 480 , in solventless zeolites, ${ }^{10}$ aqueous micelles, ${ }^{11}$ and in water pools in a sol-gel matrix ${ }^{12}$ and vesicles. ${ }^{13}$ Due to their limited time resolution they are only able to probe longer-time solvation dynamics which they found depend strongly on the environment. For example, the long-time decay is $\sim 0.8 \mathrm{~ns}$ in the sol-gel matrix, ${ }^{12} 0.6-2.4 \mathrm{~ns}$ in micelles (using different surfactants), ${ }^{11} 11 \mathrm{~ns}$ in vesicles, ${ }^{13}$ and $\sim 15.4 \mathrm{~ns}$ in the solventless zeolites. ${ }^{10}$ (The solvent relaxation dynamics for Coumarin 480 in bulk aqueous solution takes place in 310 fs. ${ }^{39}$ ) Clearly, the presence of a solvent and the constraints placed on the solvent by the structured environment strongly modify the solvation dynamics.

Levinger and co-workers ${ }^{14-20}$ have carried out timedependent fluorescence experiments on a variety of reverse micelles using Coumarin 343 as a probe molecule. They found that the solvation dynamics occur on multiple time scales and are slower than in bulk or electrolyte solutions with the longest time constant $\sim 100-350$ ps. ${ }^{15-18}$ They have investigated the effect of changing the identity or presence of the counterion, ${ }^{15,19}$ the polar solvent in the reverse micelle interior, ${ }^{16,20}$ and the surfactant molecule. ${ }^{17,18}$ They attribute the solvation dynamics to different water types present in the reverse micelle. The relative proportion of these water types depends on the water content, and hence the size, of the reverse micelle. Based on comparisons with electrolyte solutions they assert that the solvation dynamics in the reverse micelles is not governed solely by water-ion interactions but involves a significant contribution from the restricted environment. ${ }^{15,18}$

Theoretical work on confined solvents has focused primarily on the structure and dynamics of pure solvents s $^{30,34,37}$ or reverse micelle systems ${ }^{32-35}$ with only a few studies of solvation dynamics, ${ }^{31,35,36}$ most notably recent work by 
Bagchi and co-workers, ${ }^{31}$ Senapati and Chandra, ${ }^{36}$ and Faeder and Ladanyi. ${ }^{35}$

Senapati and Chandra ${ }^{36}$ were apparently the first to simulate the solvation dynamics in a nanoconfined solvent. Their system consisted of a Stockmayer fluid in a spherical nanocavity (similar to the one used here) and a LennardJones solute that is charged (excited state) or neutral (ground state). They found that the solvation dynamics in a nanocavity exhibits a similar inertial relaxation, though with a smaller amplitude, to that in the bulk solvent. In contrast, the long-time relaxation is $\sim 4$ times slower in the nanocavity than in the bulk.

More recently, Faeder and Ladanyi ${ }^{35}$ simulated timedependent fluorescence dynamics in model aqueous reverse micelles and hydrophobic cavities. Their hydrophobic cavity model ${ }^{34}$ developed by Linse and Halle, ${ }^{33}$ is the same as that used here; the reverse micelle model consists of the same cavity framework with anionic headgroups and cationic counterions added. ${ }^{34}$ The solvation dynamics were studied using an anionic diatomic probe molecule with symmetrically (ground state, $\mu=0$ ) or asymmetrically (excited state, $\mu=7.76 \mathrm{D}$ ) distributed charge. They simulated the solvation dynamics for the first 2 picoseconds after excitation and obtained results that were relatively independent of the size of the reverse micelle. In addition, the dynamics in the model reverse micelles were very similar to those in the hydrophobic cavities.

Recently, Turner et al. ${ }^{38}$ used a transition state theory approach to calculate reaction rate constants for the $\mathrm{H}_{2}+\mathrm{I}_{2}$ $\rightarrow 2 \mathrm{HI}$ reaction in confined solvents. However, they considered only one polar solvent and assumed that the reactant and transition state geometries and the activation barrier were unaffected by the solvent. As a result, their study only examined the case where the reaction was weakly coupled to the solvent and they found weak solvent effects on the reaction rate constant.

In this paper simulations of the absorption and fluorescence spectra of a solute molecule with a charge-transfer transition are presented. This represents one of the simplest and most generic examples of a process that is strongly affected by the solvent. A fundamental understanding of charge-transfer spectroscopy in nanoconfined solvents may impact many areas of chemistry by providing important insights into the larger class of solvent-coupled processes. These investigations will help to uncover some of the possible phenomena that might be observed and to determine the effect of the various cavity characteristics on reaction dynamics and spectroscopy.

The remainder of this paper is structured as follows: The model solute, solvent, and nanocavity system is described in Sec. II. The details of the application of the Monte Carlo simulation method to calculate the spectra, distribution functions, and free energies are given in Sec. III. The results of the simulations are presented and discussed in Sec. IV. Finally, some concluding remarks are offered in Sec. V.

\section{NANOCAVITY SYSTEM}

In this paper simulations of a solute dissolved in a solvent confined inside a spherical nanocavity are presented.
TABLE I. The parameters for the interaction models of the solute and solvent molecules used in the Monte Carlo simulations are given. The parameters $\epsilon$ and $\sigma$ define the Lennard-Jones interactions, $q$ the site charge, and $r_{i j}$ the distance between the site listed and the previous site.

\begin{tabular}{|c|c|c|c|c|}
\hline Site & $\epsilon(\mathrm{kcal} / \mathrm{mol})$ & $\sigma(\AA)$ & $q$ & $r_{i j}(\AA)$ \\
\hline \multicolumn{5}{|c|}{ Solute } \\
\hline \multicolumn{5}{|c|}{ Ground state } \\
\hline$A$ & 0.3976 & 3.5 & +0.1 & \\
\hline$B$ & 0.3976 & 3.5 & -0.1 & 3.0 \\
\hline \multicolumn{5}{|c|}{ Excited state } \\
\hline A & 0.3976 & 3.5 & +0.5 & \\
\hline$B$ & 0.3976 & 3.5 & -0.5 & 3.0 \\
\hline \multicolumn{5}{|c|}{ Solvents } \\
\hline \multicolumn{5}{|c|}{$\mathrm{CH}_{3} \mathrm{I}$ (Ref. 41) } \\
\hline $\mathrm{CH}_{3}$ & 0.2378 & 3.77 & +0.25 & \\
\hline I & 0.5985 & 3.83 & -0.25 & 2.16 \\
\hline \multicolumn{5}{|c|}{$\mathrm{CH}_{3} \mathrm{CN}$ (Ref. 42) } \\
\hline $\mathrm{CH}_{3}$ & 0.207 & 3.775 & +0.15 & \\
\hline $\mathrm{C}$ & 0.150 & 3.65 & +0.28 & 1.458 \\
\hline $\mathrm{N}$ & 0.170 & 3.2 & -0.43 & 1.157 \\
\hline
\end{tabular}

The solute is a model diatomic molecule (hereafter denoted as $A B$ ) with parameters adapted from a system used previously by Carter and Hynes. ${ }^{40}$ It consists of Lennard-Jones and Coulombic interactions; the model parameters are given in Table I. The Lennard-Jones parameters are the same for $A$ and $B$, and are independent of the electronic state. The two electronic states are related by a charge-transfer transition with the ground state relatively neutral $(\mu=1.44 \mathrm{D})$ and the excited state quite polar $(\mu=7.1 \mathrm{D})$. For generality the electronic structure is described in terms of a two valence-bond state model with each valence-bond state having fixed charges and the excited state $2 \mathrm{eV}$ higher in energy than the ground state. However, the electronic coupling between the valence-bond states is taken to be only $0.01 \mathrm{eV}$ so that these charges are essentially those of the ground and excited states, respectively. Thus, these simulations involve effectively fixed charges in the two electronic states.

Simulations have been carried out with $\mathrm{CH}_{3} \mathrm{I}$ and $\mathrm{CH}_{3} \mathrm{CN}$ solvents. These solvents have been chosen because the molecules are structurally similar but the dielectric constants are significantly different: $\epsilon=7$ for $\mathrm{CH}_{3} \mathrm{I}$ and $\epsilon=35$ for $\mathrm{CH}_{3} \mathrm{CN}$. Interaction potentials, consisting of Lennard-Jones and Coulombic interactions, have previously been developed for these molecules. We have used the rigid molecule models of Freitas et al. ${ }^{41}$ for $\mathrm{CH}_{3} \mathrm{I}$ and Jorgensen and Briggs ${ }^{42}$ for $\mathrm{CH}_{3} \mathrm{CN}$. In both interaction models the methyl groups are treated as a "unified atom." The parameters defining the interaction models for the solute and solvent molecules are given in Table I.

The interactions of the solute and solvent molecules with the cavity walls involve only Lennard-Jones interactions. We adopt the model developed by Linse and Halle ${ }^{33}$ and subsequently used by Faeder and Ladanyi ${ }^{34}$ in which the LennardJones interaction is averaged over the cavity surroundings. The result is a potential that depends only on the radial distance of the Lennard-Jones site on the molecule from the center of the cavity. ${ }^{33,34}$ We use the same parameters as Ref. 34: $\sigma_{\text {Wall }}=2.5 \AA, \epsilon_{\text {Wall }}=0.46 \mathrm{kcal} / \mathrm{mol}$. The cavity radius, 
TABLE II. The number of molecules, $N_{\mathrm{mol}}$, in the nanocavity is given for both the $\mathrm{CH}_{3} \mathrm{I}$ and $\mathrm{CH}_{3} \mathrm{CN}$ solvents as a function of cavity radius, $R_{\text {cav }}$. The number includes the solvent molecules and the single solute molecule.

\begin{tabular}{ccc}
\hline \hline & \multicolumn{2}{c}{$N_{\text {mol }}$} \\
\cline { 2 - 3 }$R_{\text {cav }}(\AA)$ & $\mathrm{CH}_{3} \mathrm{I}$ & $\mathrm{CH}_{3} \mathrm{CN}$ \\
\hline 10 & 16 & 16 \\
12 & 30 & 30 \\
15 & 64 & 63 \\
20 & 164 & \\
\hline
\end{tabular}

$R_{\text {cav }}$ is taken to be $10,12,15$, or $20 \AA$. In each simulation the density of the solution inside the cavity is taken to be approximately the same for a given solvent (but different for $\mathrm{CH}_{3} \mathrm{I}$ and $\mathrm{CH}_{3} \mathrm{CN}$ ). The volume used in calculating this density is obtained by reducing the nominal cavity radius by $0.5 \sigma_{\text {wall }}$ to approximately account for the excluded volume (a quantity that changes significantly with cavity size).

\section{MONTE CARLO SIMULATIONS}

Monte Carlo simulations of a single solute molecule in nanoconfined $\mathrm{CH}_{3} \mathrm{I}$ and $\mathrm{CH}_{3} \mathrm{CN}$ solvents were carried out and the results, including the absorption and fluorescence spectra, distribution functions, and free energies, are presented in Sec. IV. Solute molecules in both the ground and excited states in nanocavities of varying size were simulated, providing absorption and fluorescence spectra as well as distribution functions and free energies useful in interpreting the spectra. All simulations were carried out at a temperature of $298 \mathrm{~K}$. The absorption and fluorescence spectra are calculated by a golden rule approach, in which the spectral intensity is given by ${ }^{43}$

$$
I(\omega) \propto\left\langle\left|\mu_{\mathrm{ex}, \mathrm{gr}}\right|^{2} \delta\left(E_{\mathrm{ex}}-E_{\mathrm{gr}}-\hbar \omega\right)\right\rangle .
$$

Here $\langle\cdots\rangle$ indicates a thermal average with the solute in the ground (excited) state for the absorption (fluorescence) spectrum and $\mu_{\text {ex,gr }}$ is the transition dipole moment. The transition dipole moment is that for a two valence-bond state model, however, due to the small electronic coupling, it is essentially constant in these simulations. Thus, the spectra represent the distribution of energy gaps, $E_{\mathrm{ex}}-E_{\mathrm{gr}}$, experienced by the solute due to interactions with the surrounding solvent. (The cavity wall interactions with the solute are identical for the ground and excited states.) The primary focus here is on the position and widths of the spectra, not the relative intensities. Hence the proportionality constant implicit in Eq. (3.1) is taken such that $I\left(\omega_{\max }\right)=1$, where $\omega_{\max }$ is the frequency at which the intensity is a maximum.

The simulations in confined solvents are initiated from a bulk solvent configuration. The number of molecules, $N_{\text {mol }}$, to be enclosed in the cavity is calculated from the desired density. (Table II lists the number of molecules for each solvent and cavity size.) Then $N_{\text {mol }}$ molecules are selected from the bulk solvent configuration by including those that fall inside a spherical cavity. The center of the spherical cavity is chosen such that the solute is included, however the distance of the solute from the center is chosen randomly. Initially, the cavity radius is made large enough to include $N_{\text {mol }}$ molecules even when this is greater than the final desired radius. The radius is then reduced during a warm-up period in which it is reduced by $0.1 \AA$ every 100 cycles $\left(1\right.$ cycle $=N_{\text {mol }}$ steps $)$. Once the desired radius is reached, the equilibration continues until a total of 400000 cycles have been completed, followed by 4000000 (3000000) cycles for the $\mathrm{CH}_{3} \mathrm{I}$ $\left(\mathrm{CH}_{3} \mathrm{CN}\right)$ simulations during which data is collected.

\section{A. Free energies}

Free energy surfaces as a function of the solute position are calculated using thermodynamic integration. ${ }^{44,45}$ This approach is necessary to accurately obtain the free energy at radii where the solute molecule is infrequently found. In this approach, a Monte Carlo simulation is carried out with the solute molecule frozen at a fixed position in the cavity and the average radial force on the solute molecule center-ofmass,

$$
\left\langle F_{r}\right\rangle=\left\langle-\frac{\partial U}{\partial r}\right\rangle=\frac{\int d \mathbf{q} \int d \mathbf{p}\left(-\frac{\partial U}{\partial r}\right) e^{-\beta H(\mathbf{p}, \mathbf{q})}}{\int d \mathbf{q} \int d \mathbf{p} e^{-\beta H(\mathbf{p}, \mathbf{q})}},
$$

is calculated, where $r$ is the distance of the solute center-ofmass from the center of the spherical cavity. The Helmholtz free energy can then be obtained by integration of the average force over the radial distance,

$$
A(r)=A\left(r_{0}\right)-\int_{r_{0}}^{r} d r\left\langle F_{r}\right\rangle .
$$

In practice, the average force is calculated at $0.25 \AA$ intervals in the radial coordinate. Each Monte Carlo simulation to calculate the average force consists of 400000 equilibration cycles and 2000000 cycles over which the average is computed.

Two-dimensional free energy surfaces as a function of the solute radial coordinate and collective solvent coordinate are calculated from this same set of simulations. The solvent coordinate is defined as the difference in the ground and excited state interaction energies between the solute and the solvent. Since the only difference between the ground and excited state interactions of the solute molecule with the solvent molecules is due to the different charges, the solvent coordinate can be calculated as

$$
\Delta E=E_{\mathrm{ex}}-E_{\mathrm{gr}},
$$

where $E_{\mathrm{gr}}$ and $E_{\mathrm{ex}}$ are the ground and excited state energies. Thermodynamic integration is not used to compute the free energy as a function of the solvent coordinate. Rather, a straightforward histogram approach is used. For each simulation with a solute molecule fixed at a specific value of $r$, required for the thermodynamic integration discussed above, we histogram the occurrences of the solvent coordinate values. This gives a distribution function of the solvent coordinate, $P(\Delta E ; r)$, from which the free energy can be obtained as

$$
\Delta A(\Delta E ; r)=-k_{B} T \ln P(\Delta E ; r) .
$$



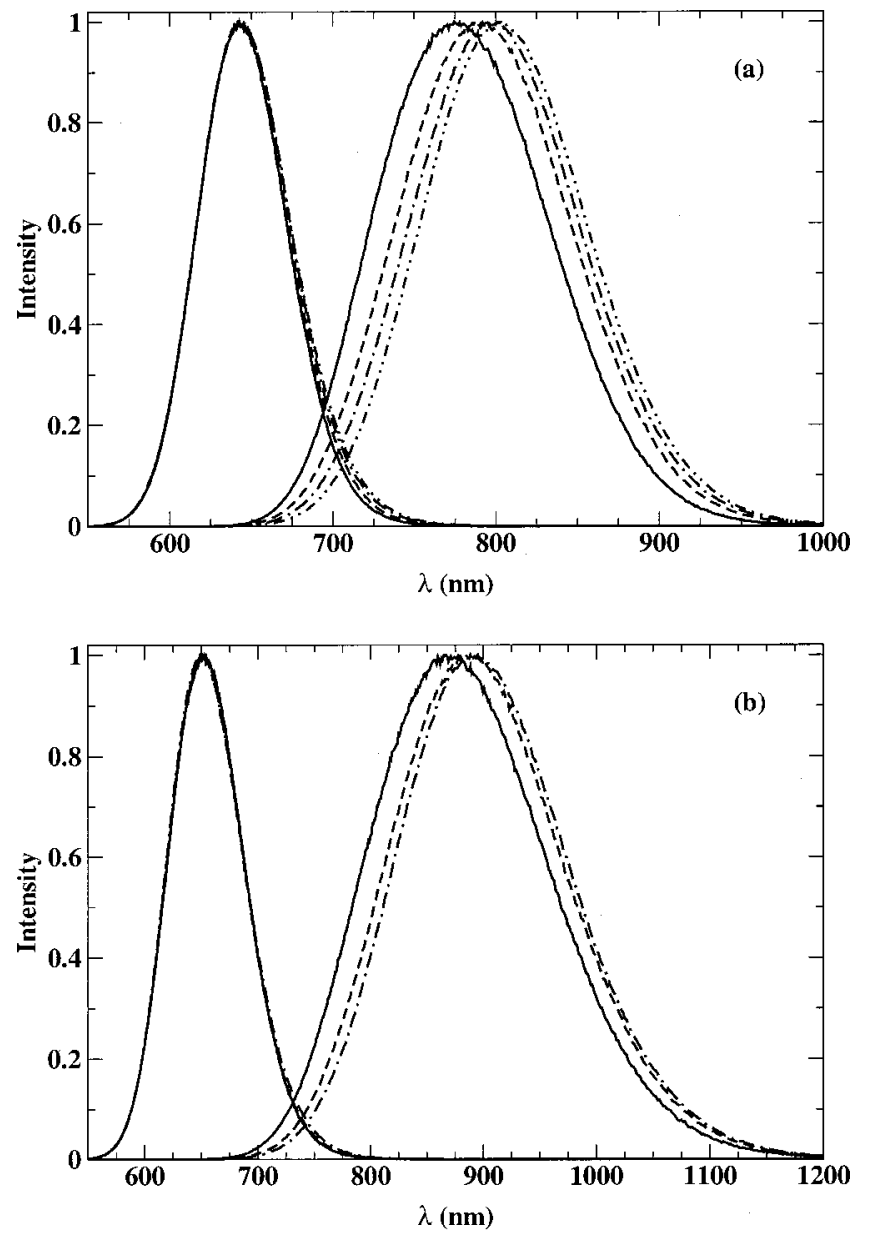

FIG. 1. The calculated absorption and fluorescence spectra for a solute molecule in (a) $\mathrm{CH}_{3} \mathrm{I}$ (density, $\rho=1.4 \mathrm{~g} / \mathrm{cm}^{3}$ ) and (b) $\mathrm{CH}_{3} \mathrm{CN}$ (density, $\rho=0.4 \mathrm{~g} / \mathrm{cm}^{3}$ ) are shown for nanocavities of different sizes. The spectra shown are for solute molecules in cavities of radius $10 \AA$ (solid line), $12 \AA$ (dashed line), $15 \AA$ (dot-dashed line), and $20 \AA$ (dot-dot-dashed line, $\mathrm{CH}_{3} \mathrm{I}$ only).

This approach only allows an accurate calculation of the free energy surface near the equilibrium value of the solvent coordinate.

\section{RESULTS AND DISCUSSION}

\section{A. Absorption and fluorescence spectra}

\section{1. $\mathrm{CH}_{3}$ l solvent}

The absorption and fluorescence spectra for a single solute molecule in $\mathrm{CH}_{3} \mathrm{I}$ solvent in cavities of radius $R_{\text {cav }}$ $=10,12,15$, and $20 \AA$ are shown in Fig. 1(a). Clearly, the absorption and fluorescence spectra have distinctly different behavior as a function of the nanocavity size. The fluorescence spectrum shifts consistently to the red (longer wavelengths) with increasing cavity radius. The fluorescence maximum is $\lambda_{\max }=776,790,798$, and $804 \mathrm{~nm}$ for $R_{\text {cav }}$ $=10,12,15$, and $20 \AA$, respectively. In contrast, the absorption spectrum changes little with increasing cavity radius; the absorption maximum is within $1 \mathrm{~nm}$ of $\lambda_{\max }=643 \mathrm{~nm}$ for all cavity radii. The width of the absorption spectrum is also essentially independent of the size of the nanocavity, the full width at half maximum is $\sim 65 \mathrm{~nm}$ for all cavity radii. The fluorescence spectra are significantly broader than the absorption spectra but the widths are also fairly independent of cavity size at $\sim 123 \mathrm{~nm}$.

The fluorescence spectra are consistent with the intuitive idea that the solvent polarity increases with the cavity size and the number of solvent molecules. The dependence of the solvent polarity on the cavity size was calculated in a previous theoretical study by Senapati and Chandra ${ }^{37}$ of the dielectric constant of confined water in similar spherical nanoscale cavities. Using the SPC/E water model for which the bulk dielectric constant is $\epsilon=72$, they found that the dielectric constant increased from $\epsilon=39$ for a $6.1 \AA$ radius cavity to $\epsilon=60$ for a $12.2 \AA$ radius cavity. ${ }^{37}$ Thus, making the cavity bigger is analogous to increasing the solvent polarity in a bulk system. The shifts in the fluorescence spectrum as the cavity radius is increased can therefore be attributed to the better solvation associated with a larger, and perhaps less constrained, solvent pool. This argument should apply equally well to the absorption spectrum yet this is not observed. Additional information is necessary to understand this difference between the absorption and fluorescence spectra as discussed in Sec. IV B.

\section{2. $\mathrm{CH}_{3} \mathrm{CN}$ solvent}

The absorption and fluorescence spectra for a solute molecule in $\mathrm{CH}_{3} \mathrm{CN}$ solvent are shown for $R_{\text {cav }}=10,12$, and $15 \AA$ in Fig. 1(b). The dependence of the absorption and fluorescence spectra on the cavity radius is qualitatively the same as for the $\mathrm{CH}_{3} \mathrm{I}$ solvent. Specifically, the fluorescence spectrum shifts to longer wavelengths with increasing cavity size while the absorption spectrum is hardly affected. The maximum in the fluorescence spectrum occurs at $\lambda_{\max }$ $=869,888$, and $893 \mathrm{~nm}$ for $R_{\mathrm{cav}}=10,12$, and $15 \AA$, respectively. The maximum in the absorption spectra is within 1 $\mathrm{nm}$ of $\lambda_{\max }=650 \mathrm{~nm}$ for each cavity radius. Thus, the solvent polarity does not qualitatively affect the spectra. The primary quantitative difference is that the spectra are shifted to longer wavelengths, by $\sim 7$ and $\sim 100 \mathrm{~nm}$ for the absorption and fluorescence spectra, respectively. This result is the same as would be expected for bulk solvents as the polarity is increased. The spectra of the solute in $\mathrm{CH}_{3} \mathrm{CN}$ are also broader than those in the $\mathrm{CH}_{3} \mathrm{I}$ solvent, with absorption and fluorescence spectrum full widths at half maximum of $\sim 80$ and $\sim 180 \mathrm{~nm}$, respectively.

\section{B. Solute radial densities}

\section{1. $\mathrm{CH}_{3}$ l solvent}

The probability distributions for the solute molecule center-of-mass position are shown in Fig. 2 as a function of the distance from the cavity wall. Distributions are given for the molecule in its ground and excited states and for cavities of radius $10,12,15$, and $20 \AA$. These distributions are normalized so that the integral of the probability distribution over all radial distances (i.e., the probability of finding the solute molecule in the cavity) is one. It is important to note that these distributions are representative of the solute positions that contribute to the absorption and fluorescence spectra. It is for this reason that we plot the probability distribu- 

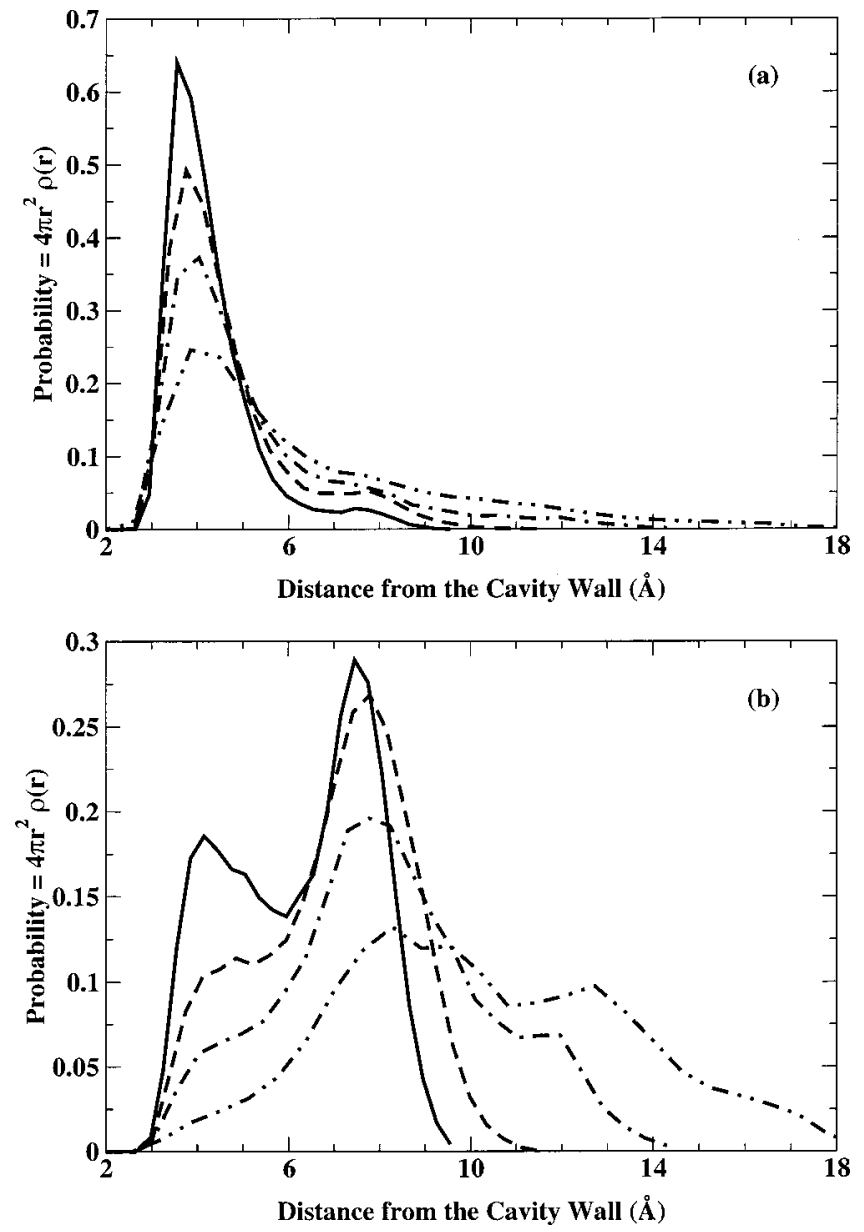

FIG. 2. The radial probability density is plotted for a solute molecule in the (a) ground and (b) excited state in nanocavities of different size. Results are shown for a solute molecule in $\mathrm{CH}_{3} \mathrm{I}\left(\rho=1.4 \mathrm{~g} / \mathrm{cm}^{3}\right)$ in cavities of radius 10 $\AA$ (solid line), $12 \AA$ (dashed line), $15 \AA$ (dot-dashed line), and $20 \AA$ (dotdot-dashed line)

tion rather than the radial density, $\rho(r)$. Note that the fall-off in the distributions at large distances from the wall, i.e., those approaching $R_{\mathrm{cav}}$, is due to the decrease in the available volume for the solute molecule.

Focusing first on the probability distributions for the solute in its ground electronic state, shown in Fig. 2(a), the most probable solute molecule position is near the cavity wall, independent of the cavity radius. However, the distribution is broader for larger cavity radii, indicating that the probability of finding a solute molecule away from the wall increases with cavity size. It is important to note that there are two effects that contribute to this probability distribution: the space available for the solute molecule at a given distance from the center of the cavity (which is proportional to $4 \pi r^{2}$ ) and the dependence of the free energy on the solute position (considered in Sec. IV D). Obviously, there is more space available for the solute molecule near the cavity wall than near the center of the cavity and this contributes to the large probability of finding the solute molecule near the wall. However, as discussed in Sec. IV D free energy considerations play a role here as well.

The probability distributions for the solute molecule in its excited electronic state, shown in Fig. 2(b), are distinctly different than those for the ground state. The most probable location of a solute molecule in the excited state is in the interior of the cavity. As the cavity size is increased, the most probable position for the solute moves farther from the wall and the distribution broadens. For the smaller cavities, $R_{\text {cav }}$ $=10$ and $12 \AA$, there is still a significant probability of finding the solute near the cavity wall. However, this probability decreases as the cavity size increases and for a $20 \AA$ radius cavity is quite small. Since the interactions of the solute in its ground and excited state are the same except for the atomic charges, the differences in the probability distributions between the electronic states can only be due to electrostatic effects. The large charges of the excited state solute molecule are better solvated in the cavity interior, surrounded by a complete shell of solvent, than near the cavity wall.

The different dependence of the absorption and fluorescence spectra on the cavity size can be attributed to the distinctly different distributions of solute positions in the ground and excited states. Since the most probable solute position is against the cavity wall in the ground state its environment, consisting of only a partial solvation shell, does not change much with $R_{\text {cav }}$. This is reflected in the absorption spectrum which is effectively independent of the cavity radius. On the other hand, the solute is primarily in the cavity interior in the excited state and the position distribution shifts further from the wall as $R_{\text {cav }}$ increases. This shifting of the distribution and the increasing effective solvent polarity with increasing cavity radius ${ }^{37}$ lead to a consistent redshift in the fluorescence spectrum.

\section{2. $\mathrm{CH}_{3} \mathrm{CN}$ solvent}

The probability distributions for the solute molecule position as a function of distance from the cavity wall are shown in Fig. 3 for $R_{\text {cav }}=10,12$, and $15 \AA$. These distributions are qualitatively the same as for a solute molecule in $\mathrm{CH}_{3} \mathrm{I}$ solvent. Specifically, the ground state probability distribution, shown in Fig. 3(a), is sharply peaked near the cavity wall for all cavity sizes. The distribution becomes broader and the maximum shifts slightly to longer distances as the cavity radius is increased.

The probability distributions for the excited solute, shown in Fig. 3(b), indicate that the molecule is most likely to be found in the interior of the cavity independent of the radius. As for the $\mathrm{CH}_{3} \mathrm{I}$ results, the distribution broadens and the probability of finding the solute near the wall decreases as $R_{\text {cav }}$ increases. In addition, there is a slight shift in the position of the largest peak toward greater distances from the wall.

Based on the comparison of the simulation results with these two solvents with roughly equal numbers of molecules in the cavity, the solvent polarity has a very small effect on the solute radial probability distributions. There are no qualitative differences and the quantitative differences are minor. The effect of the solvent polarity is discussed further in Sec. IV D where the free energy as a function of the solute position in the cavity is considered. 

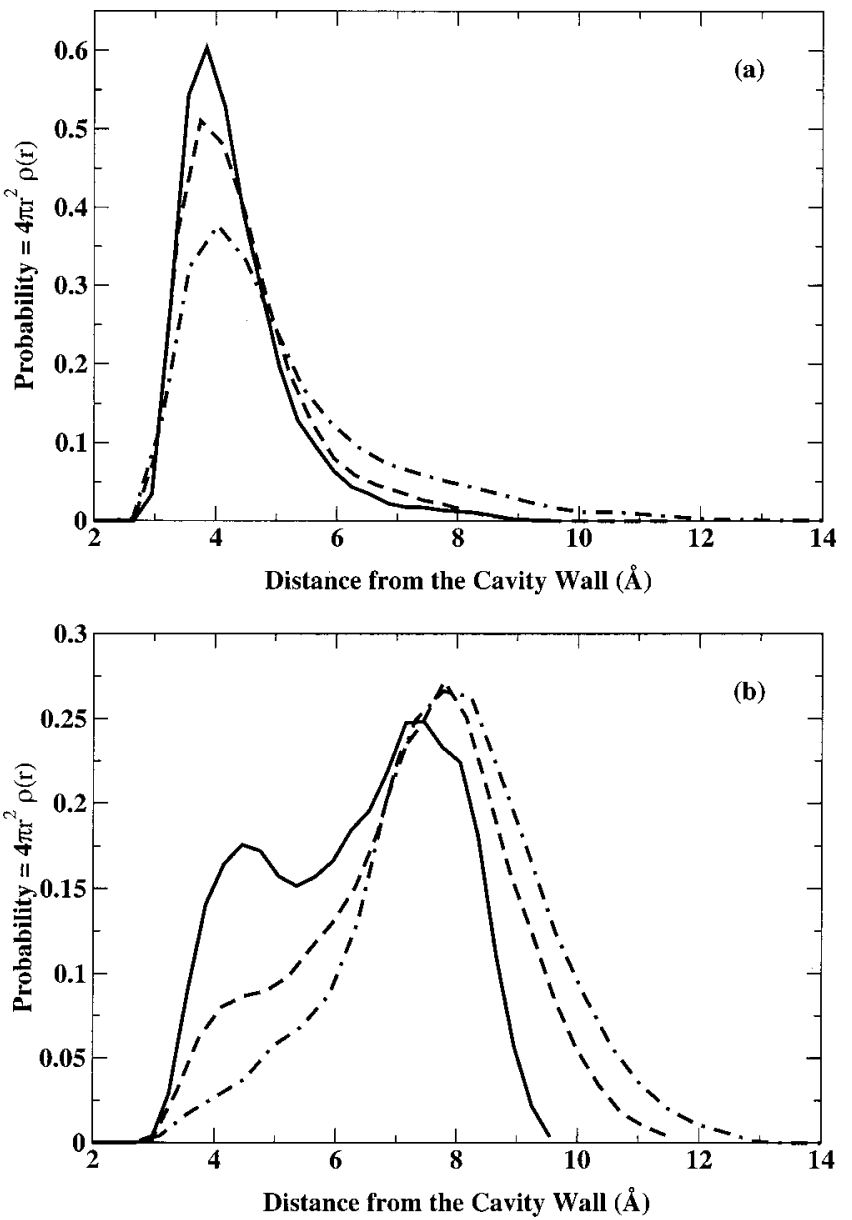

FIG. 3. Same as Fig. 2 but for $\mathrm{CH}_{3} \mathrm{CN}$ solvent $\left(\rho=0.4 \mathrm{~g} / \mathrm{cm}^{3}\right)$. (No results are shown for $R_{\text {cav }}=20 \AA$.)

\section{Solute angular distributions}

The probability distributions for the angle of the solute molecule bond with the surface normal of the cavity wall are shown in Fig. 4. The distributions are shown as a function of $\cos \theta$ for the solute in the ground and excited electronic states in $\mathrm{CH}_{3} \mathrm{I}$ solvent in cavities of radius $10,12,15$, and 20 $\AA$. The angle $\theta$ is that between the vector from the molecule center-of-mass to the nearest point on the cavity wall and the $A-B$ vector. It is defined such that $\cos \theta=1(\cos \theta=-1)$ corresponds to the $A B$ molecule perpendicular to the cavity wall with $B(A)$ nearest the wall. The solute bond parallel to the wall corresponds to $\cos \theta=0$.

For a solute molecule in the ground state the angular probability distribution is centered about $\cos \theta=0$, indicating that the most probable orientation is parallel to the cavity wall. This result is consistent with the radial probability distributions discussed in Sec. IV B in that a solute molecule near the cavity wall (the most likely position in the ground state) would be expected to lie parallel to the uncharged wall. The angular distribution is slightly asymmetric with a small preference for the negatively charged $B$ to be closer to the wall. In addition, the angular distribution broadens as the cavity radius is increased. This is also consistent with the radial distributions which broaden as the cavity size is increased. Thus the angular distributions shown in Fig. 4(a)
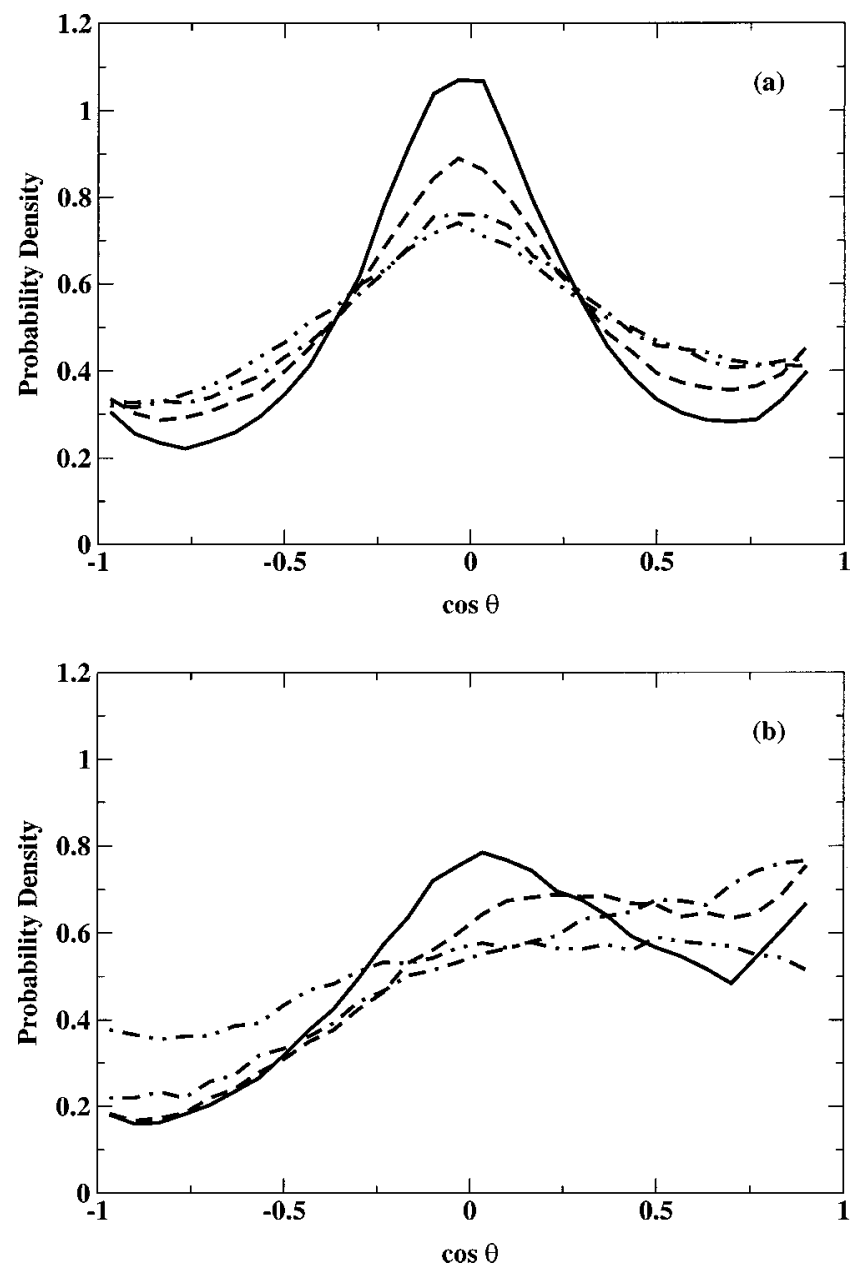

FIG. 4. The angular probability density is plotted for a solute molecule in the (a) ground and (b) excited state in nanocavities of different size. See the text for the definition of the angle $\theta$. Results are shown for a solute molecule in $\mathrm{CH}_{3} \mathrm{I}\left(\rho=1.4 \mathrm{~g} / \mathrm{cm}^{3}\right)$ in cavities of radius $10 \AA$ (solid line), $12 \AA$ (dashed line), $15 \AA$ (dot-dashed line), and $20 \AA$ (dot-dot-dashed line).

reflect the greater probability in larger cavities of finding the solute in the cavity interior and indicate that in the cavity interior any orientational preference is at best significantly weaker.

The angular distributions for the excited state solute molecule, shown in Fig. 4(b), are more uniform than those for the ground state. A distribution for a molecule with no preferential orientation would be a constant value of 0.5 for all $\cos \theta$ values and this limit is approached for $R_{\text {cav }}=20 \AA$. The distributions for all cavity sizes indicate that the orientation of the solute molecule is more likely to have the negatively charged $B$ atom pointing toward the cavity wall. This preferential orientation becomes less pronounced as the cavity size increases. In contrast to the distributions for the ground state solute molecule, only for the $10 \AA$ cavity is there a significant peak at $\cos \theta=0$. For this cavity radius, the most probable solute molecule orientation is parallel to the wall. This is a reflection of the greater likelihood of finding the solute molecule near the wall for $R_{\text {cav }}=10 \AA$, as shown in Fig. 2(b). 

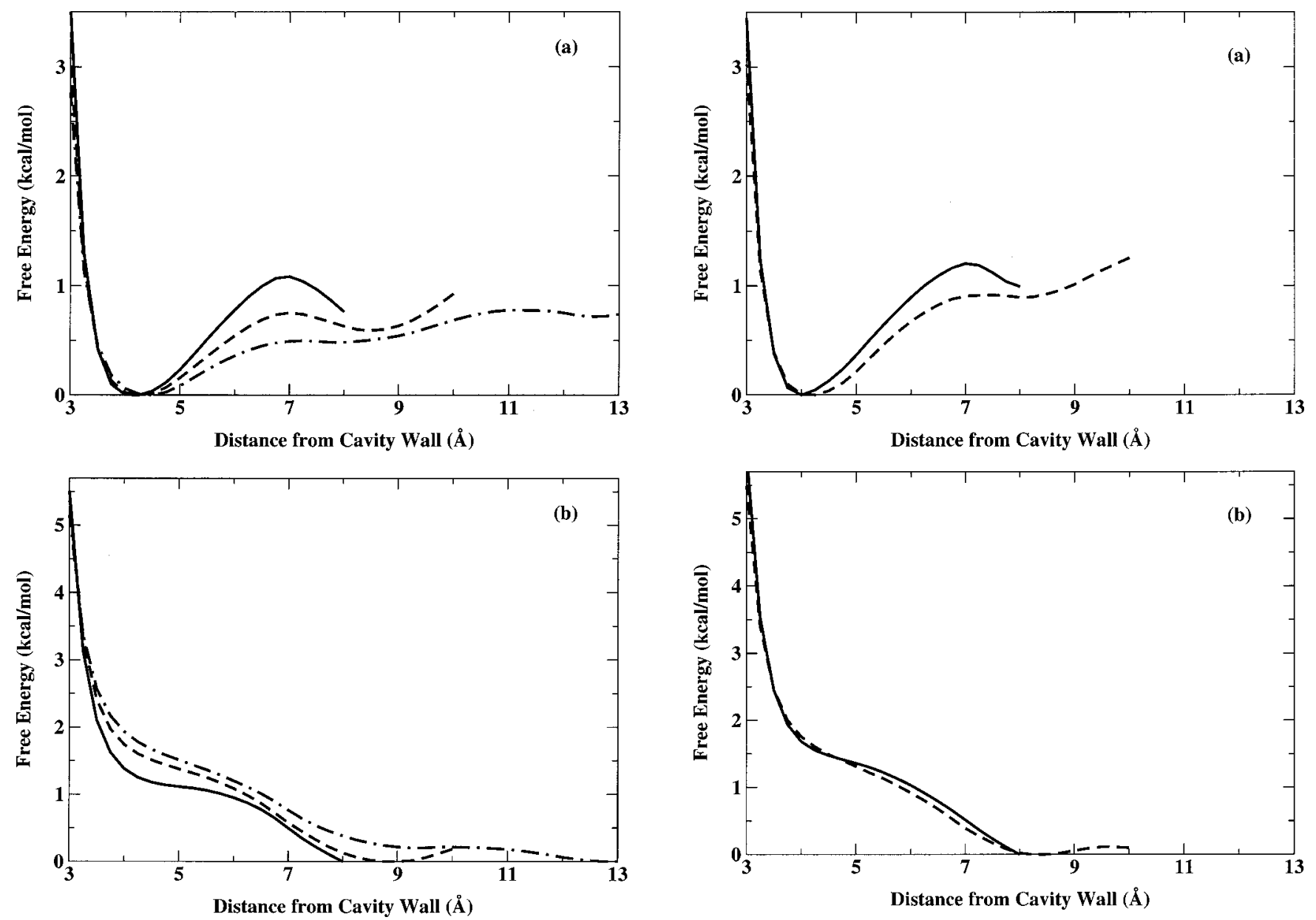

FIG. 5. The radial free energy is plotted for a solute molecule its (a) ground and (b) excited state in nanocavities of different size. Results are shown for a solute molecule in $\mathrm{CH}_{3} \mathrm{I}\left(\rho=1.4 \mathrm{~g} / \mathrm{cm}^{3}\right)$ in cavities of radius $10 \AA$ (solid line), $12 \AA$ (dashed line), and $15 \AA$ (dot-dashed line).

\section{Free energy versus solute position}

The Helmholtz free energy as a function of the distance of the solute center-of-mass from the cavity wall in $\mathrm{CH}_{3} \mathrm{I}$ solvent is shown in Fig. 5 for $R_{\text {cav }}=10,12$, and $15 \AA$. Analogous free energy curves are shown in Fig. 6 for the $\mathrm{CH}_{3} \mathrm{CN}$ solvent for $R_{\text {cav }}=10$ and $12 \AA$. Results are presented for both ground and excited state solute molecules. The minimum in each free energy curve has been set to zero for purposes of comparison.

As noted in Sec. III A, the free energies presented here are calculated by thermodynamic integration. The free energies can also be calculated from the probability distributions shown in Figs. 2 and 3 in a manner analogous to that used for the solvent coordinate [see Eq. (3.5) and Sec. IV E]. The free energy as a function of the solute position obtained in this way is in good agreement with the results obtained by thermodynamic integration except for positions where the probability of finding the solute is small (e.g., in the ground state at large distances from the cavity wall). The sampling in these regions is insufficient to accurately obtain the free energy, thus we have used thermodynamic integration which does not suffer from this difficulty.

FIG. 6. The same as Fig. 5 but for the $\mathrm{CH}_{3} \mathrm{CN}$ solvent $\left(\rho=0.4 \mathrm{~g} / \mathrm{cm}^{3}\right)$. (No results are shown for $R_{\text {cav }}=15 \AA$.)

\section{Ground state}

For the ground state solute molecule the position of the global minimum in the free energy surface is relatively independent of the cavity size, occurring at a distance of $\sim 4.25$ $\AA$ from the cavity wall for $R_{\text {cav }}=10$ and $12 \AA$ and at $\sim 4.5 \AA$ for $R_{\text {cav }}=15 \AA$. There is a free energy barrier, or local maximum, for the solute to move into the interior of the cavity at $\sim 7 \AA$ for $R_{\text {cav }}=10$ and $12 \AA$ and at $\sim 7.25 \AA$ for $R_{\text {cav }}$ $=15 \AA$. The height of this barrier decreases with the cavity size and is $\sim 1.1,0.75$, and $0.5 \mathrm{kcal} / \mathrm{mol}$ for $R_{\mathrm{cav}}=10,12$, and $15 \AA$, respectively. This barrier and the undulations in the free energy curves at large distances reflect the ordering of the solvent into layers near the solvent-cavity wall interface.

The small charges on the ground state solute molecule mean that it has a smaller dipole moment than a solvent molecule. As a consequence, the free energy is lowest when the less polar solute is near the hydrophobic (uncharged) wall and only solvent molecules are in the cavity interior. That is, the solvent prefers to solvate another solvent molecule in the cavity interior rather than the solute molecule. As illustrated in Figs. 2 and 5 this preference becomes weaker as the cavity radius is increased. Even for the smallest cavity the free energy difference for a solute molecule near the wall versus in the cavity interior is less than $1 \mathrm{kcal} /$ 
mol, not a large difference given that $k_{B} T \sim 0.6 \mathrm{kcal} / \mathrm{mol}$. The strongly peaked probability distributions shown in Fig. 2 therefore can be attributed to a cooperation between the energetic effects and the available space at a given radial position in the cavity.

The free energy curves for the ground state solute molecule in the $\mathrm{CH}_{3} \mathrm{CN}$ solvent shown in Fig. 6(a) are qualitatively the same as the $\mathrm{CH}_{3} \mathrm{I}$ solvent results. Relatively small quantitative differences exist in the position of the global minimum and the height of the barrier to movement of the solute into the cavity interior. The free energy is a minimum at a distance of $\sim 4.0$ and $4.25 \AA$ for the 10 and $12 \AA$ radius cavities, respectively. This is $0.25 \AA$ closer to the wall than in the $\mathrm{CH}_{3} \mathrm{I}$ solvent for the $10 \AA$ radius cavity and the same for the $12 \AA$ cavity. The barrier occurs at $\sim 7.0$ and $7.5 \AA$ with a height of 1.2 and $0.9 \mathrm{kcal} / \mathrm{mol}$ for $R_{\text {cav }}=10$ and $12 \AA$, respectively. This is $0.1-0.15 \mathrm{kcal} / \mathrm{mol}$ higher than in the $\mathrm{CH}_{3} \mathrm{I}$ solvent. These results indicate that for a ground state solute molecule the primary effect of the solvent polarity is to hold the molecule more tightly to the wall. However, the energy differences involved are relatively small despite the large change in solvent polarity ( $\epsilon=7$ for $\mathrm{CH}_{3} \mathrm{I}$ and $\epsilon=35$ for $\mathrm{CH}_{3} \mathrm{CN}$ ).

\section{Excited state}

The minimum in the free energy for the excited state molecule occurs in the cavity interior for every cavity radius considered as can be seen in Fig. 5(b). The minimum for the $R_{\text {cav }}=10 \AA$ cavity occurs at the largest distance from the wall for which we have calculated the free energy, 8.0 A. For the 12 and $15 \AA$ radius cavities, the global minima occur at 9.0 and $12.75 \AA$, respectively, though the free energy curve is relatively flat in the cavity interior. In sharp contrast to the ground state free energy curves there is a significantly unfavorable free energy for the solute near the cavity wall which increases with $R_{\text {cav }}$. The energy is lower for the solute in the cavity interior relative to near the cavity wall by $\sim 1.2,1.5$, and $1.7 \mathrm{kcal} / \mathrm{mol}$ for $R_{\mathrm{cav}}=10,12$, and $15 \AA$, respectively. The dipole moment of the solute in the excited state is significantly larger than the dipole moment of a solvent molecule. Thus, the excited state solute with a large dipole moment is best solvated in the interior where it is surrounded by a full solvent shell. It is more favorable for the solvent to solvate the excited state solute than a solvent molecule. The probability distributions shown in Figs. 2(b) and 3(b) are the result of competition between the free energy considerations and the $4 \pi r^{2}$ factor accounting for the available space.

As for the ground state solute results, the free energy curves are qualitatively the same for the $\mathrm{CH}_{3} \mathrm{I}$ and $\mathrm{CH}_{3} \mathrm{CN}$ solvents. The free energy in the $\mathrm{CH}_{3} \mathrm{CN}$ solvent shown in Fig. 6(b) is only weakly dependent on the cavity radius. It has a global minimum in the interior of the cavity for both $R_{\text {cav }}=10$ and $12 \AA$ and a significant barrier for the solute to be at the cavity wall. This barrier is $\sim 1.5 \mathrm{kcal} / \mathrm{mol}$ for both cavity radii shown; this is $0.3 \mathrm{kcal} / \mathrm{mol}$ higher than for the $\mathrm{CH}_{3} \mathrm{I}$ solvent for $R_{\text {cav }}=10 \AA$ and roughly the same for the two solvents for $R_{\mathrm{cav}}=12 \AA$. Thus, the higher polarity of the $\mathrm{CH}_{3} \mathrm{CN}$ solvent results in a greater preference for the excited state solute to be in the cavity interior but this polarity effect
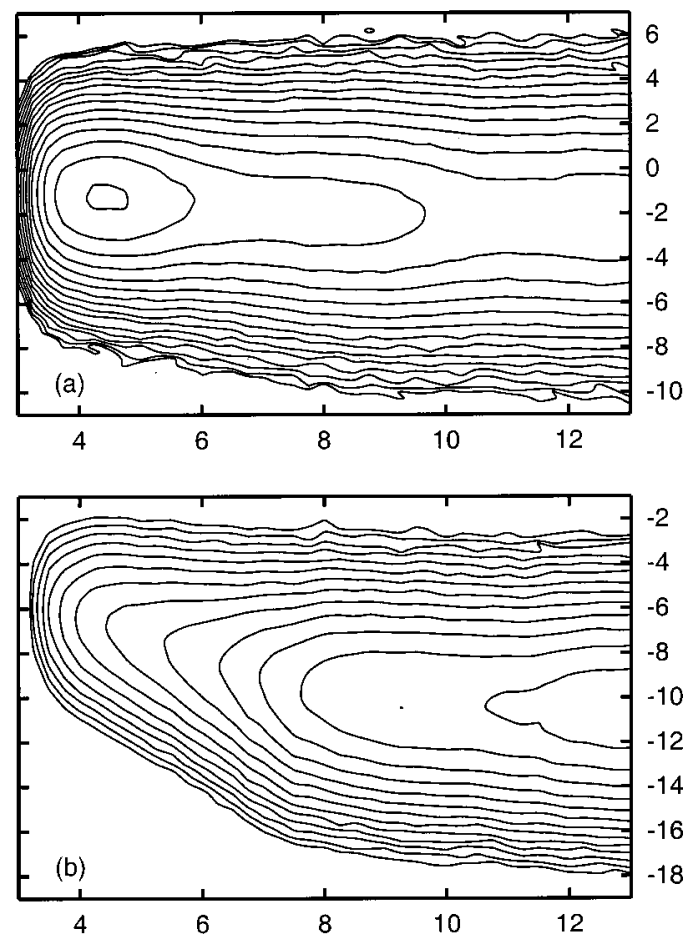

FIG. 7. Contour plots of the two-dimensional free energy surfaces are shown for the solute molecule in the (a) ground and (b) excited state in the $\mathrm{CH}_{3} \mathrm{I}$ solvent with $R_{\text {cav }}=15 \AA$. The horizontal axis is the distance of the solute center-of-mass from the cavity wall in $\AA$ and the vertical axis is the solvent coordinate, $\Delta E$, in $\mathrm{kcal} / \mathrm{mol}$. The spacing between contours is 0.3 $\mathrm{kcal} / \mathrm{mol}$.

becomes less pronounced for larger cavity radii. This effect can also be seen in the solute radial probability distributions shown in Figs. 2(b) and 3(b).

\section{E. Free energy surfaces}

Two-dimensional free energy surfaces as a function of the solute distance from the cavity wall and the collective solvent coordinate are shown as contour plots in Fig. 7. These free energy surfaces present a more complete picture of the system than can be obtained from one-dimensional plots of the free energy as a function of the solvent coordinate alone. This is particularly important in attempting to infer something about the time-dependent fluorescence based on the present results. Note that the position of the free energy minimum changes between the ground and excited states in both the solute position (as seen in Sec. IV D) and the solvent coordinate. Thus, upon excitation from the ground state both the solvent coordinate and the solute position will have nonequilibrium values in the excited state. The subsequent relaxation dynamics would then be expected to involve the usual solvent reorientation and movement of the solute toward the interior of the cavity. We would expect that the solvent reorientation dynamics should occur on a significantly faster time scale than the solute motion (which will be diffusive in nature). This would lead to solvation dynamics occurring on multiple time scales; this is a common observation in experimental studies of solvation dynamics in confined solvents. However, to our knowledge no solvation dynamics have previously been associated with the diffusive 
movement of the solute in either experimental or theoretical studies. We are currently carrying out molecular dynamics simulations of the time-dependent fluorescence to verify this prediction. Whether this phenomenon is occurring in confined solvent systems that have been or are being investigated experimentally is unclear at present. However, even if this is not the case, one could imagine designing confined solvent/solute systems that will exhibit such behavior. One caveat is that the solute position distributions depend on the geometry of the nanocavity (e.g., cylindrical versus spherical), an effect that we are currently investigating.

It is useful to discuss this prediction in the context of the two previous simulations of time-dependent fluorescence in nanoconfined solvents. Senapati and Chandra chose an atomic solute with an electronic transition that involved the creation of a charge. ${ }^{36}$ It seems likely that they would have observed similar behavior to that predicted here if they had not fixed the position of the solute near the cavity wall in their simulations. Faeder and Ladanyi simulated the timedependent fluorescence of a model solute quite similar to that used here, including a charge-transfer transition with a comparable change in the dipole moment. ${ }^{35}$ However, their solute was an anion and therefore had strong interactions with the solvent in both the ground and excited states. Hence, they did not observe a significant dependence of the distribution of solute positions on the electronic state.

It is apparent from Fig. 7 that the equilibrium value of the solvent coordinate depends strongly on the solute position in the excited state. This should also affect the relaxation dynamics probed in time-dependent fluorescence experiments. In the ground state the shift is significantly smaller. Furthermore, the force constant for motion in the solvent coordinate also appears to depend upon the solute position. This is verified by taking slices through the free energy surface along the solvent coordinate for different solute positions. Three such slices are presented for the ground and excited state in Fig. 8. The calculated free energies are indicated by the symbols and quadratic fits to the data are shown as lines. In all cases the quadratic fits describe the calculated free energy curves well. From these quadratic fits we can extract a force constant in the solvent coordinate that depends on the solute position, $k_{s}(d)$ :

$$
\Delta A(\Delta E, d)=\Delta A_{0}(d)+\frac{1}{2} k_{s}(d)\left[\Delta E-\Delta E_{0}(d)\right]^{2},
$$

where $d=R_{\text {cav }}-r$ is the distance of the solute center-of-mass from the cavity wall. This force constant is a measure of how tightly the solvent coordinate is held to its equilibrium value or alternatively, the difficulty for the solvent to undergo a fluctuation away from equilibrium with the solute charges. Hence, larger values of $k_{s}$ indicate more constrained solvent interactions with the solute.

The solvent force constants obtained for the ground state solute molecule are $k_{s}(d=4.5 \AA)=0.179(\mathrm{kcal} / \mathrm{mol})^{-1}$, $k_{s}(d=8.0 \AA)=0.133(\mathrm{kcal} / \mathrm{mol})^{-1}, \quad$ and $\quad k_{s}(d=11.5 \AA)$ $=0.113(\mathrm{kcal} / \mathrm{mol})^{-1}$. For the excited state solute, the force constant has values of $k_{s}(d=4.5 \AA)=0.176(\mathrm{kcal} / \mathrm{mol})^{-1}$, $k_{s}(d=8.0 \AA)=0.132(\mathrm{kcal} / \mathrm{mol})^{-1}, \quad$ and $\quad k_{s}(d=11.5 \AA)$ $=0.134(\mathrm{kcal} / \mathrm{mol})^{-1}$. Clearly the force constant does depend upon the position of the solute in the cavity: $k_{s}(d)$
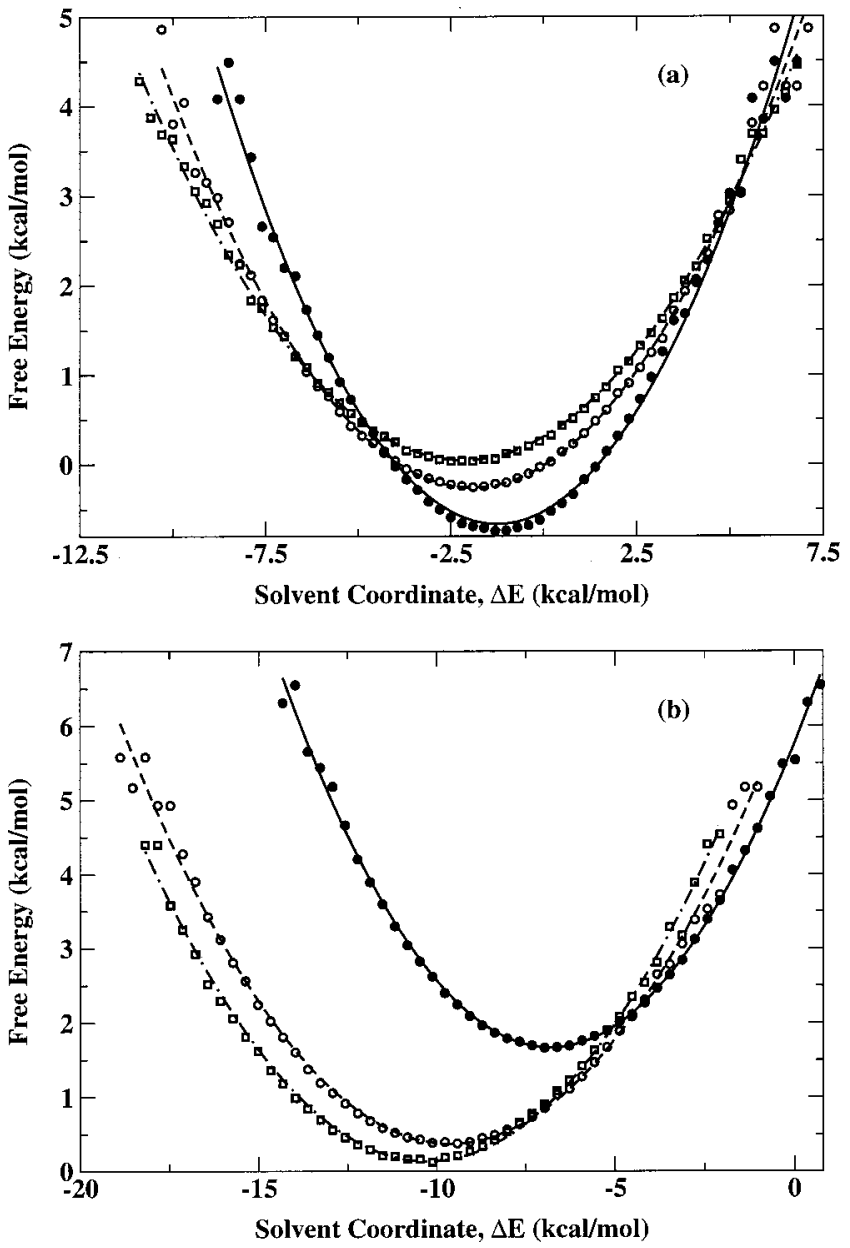

FIG. 8. The free energy as a function of the solvent coordinate is shown for the solute molecule in the (a) ground and (b) excited state in $\mathrm{CH}_{3} \mathrm{I}$ solvent with $R_{\text {cav }}=15 \AA$. Results are shown for fixed solute center-of-mass positions of $4.5 \AA$ (solid line/solid circles), $8.0 \AA$ (dashed line/open circles), and 11.5 $\AA$ (dot-dashed line/open squares) from the cavity wall. The lines represent quadratic fits to the calculated results indicated by the symbols.

increases as $d$ decreases. The values for the ground and excited states are virtually the same except for $d=11.5 \AA$ where $k_{s}$ is larger in the excited state. In fact, for the excited state $k_{s}(d)$ appears to have reached its minimum value by $d=8.0 \AA$ whereas for the ground state $k_{s}(d)$ continues to decrease as $d$ increases.

These results for the dependence of the solvent force constant as a function of the solute position suggest that the solvent molecules that surround the solute when it is near the cavity wall are more constrained due to interactions with the cavity surface. Naturally, these constraints are more weakly felt in the cavity interior where the behavior may be expected to be more like that in a bulk solvent. Previously, Carter and Hynes ${ }^{40}$ observed in bulk solvent simulations that the solvent force constant is larger in the excited state than in the ground state for a charge-transfer transition. The present force constants at $d=11.5 \AA$ are consistent with this result. At distances closer to the wall the constraints imposed by the cavity surface appear to dominate since no difference is observed in the force constants in the ground and excited states. 


\section{CONCLUDING REMARKS}

We have carried out simulations of the absorption and fluorescence spectra of a model diatomic solute with a charge-transfer transition in spherical hydrophobic nanocavities. The absorption and fluorescence spectra have a different dependence on the cavity size. The fluorescence spectrum is redshifted as the cavity radius is increased while the absorption is essentially unchanged. This behavior is attributed to the difference in the distribution of solute molecule positions probed in the absorption and fluorescence spectra. In the less polar ground state the solute is more likely to be found near the cavity wall whereas in the polar excited state the most probable solute position is in the cavity interior.

The probability distributions of the solute position are determined by two factors: the energetics and the available space. We have separated these contributions by calculating the free energy as a function of the solute center-of-mass position. For a ground state solute the free energy is a minimum near the cavity wall, while for an excited state solute it is a minimum in the cavity interior. The space available for a solute molecule a distance $r$ from the center of the cavity is proportional to $4 \pi r^{2}$ for the spherical nanocavities considered here. Thus, the two factors cooperate for a ground state solute leading to position distributions strongly peaked near the cavity wall. In contrast, the two factors compete in the case of an excited state solute with free energy considerations favoring positions in the cavity interior while the available space for the solute is greatest near the wall.

The effect of solvent polarity has been examined by the comparison of simulations with $\mathrm{CH}_{3} \mathrm{I}$ solvent $(\epsilon=7)$ and $\mathrm{CH}_{3} \mathrm{CN}$ solvent $(\epsilon=35)$. The absorption and fluorescence spectra of a solute in $\mathrm{CH}_{3} \mathrm{CN}$ are shifted to the red of those with $\mathrm{CH}_{3} \mathrm{I}$ solvent. However, we found no qualitative differences in the shifts in the spectra with $R_{\text {cav }}$, in the solute distribution functions, or in the free energy as a function of solute position. In addition, the quantitative differences are relatively small.

Free energy surfaces as a function of the solute position and a collective solvent coordinate have been calculated and exhibit differences in the equilibrium positions of both coordinates between the ground and excited states. Based on this, we predict that the solvation dynamics will involve not only solvent reorientational motion but also physical movement of the solute from near the cavity wall toward the cavity interior. To our knowledge this is the first time such a phenomenon has been suggested. Molecular dynamics simulations are currently underway to test this prediction.

The two-dimensional free energy surfaces were also used to calculate the force constant in the solvent coordinate as a function of the solute position. This force constant increases as the solute distance from the cavity wall decreases indicating that the solvent is less constrained in the cavity interior than near the cavity surface. This result has implications for how solvent-driven reactions might proceed in nanoconfined solvents, e.g., electron or proton transfer reactions for which the reaction coordinate is a solvent coordinate analogous to the one used here. These effects will be examined in future work.

The diversity among nanocavities (e.g., in their size, shape, dimensionality, flexibility, and interactions with the solvent, solute, and/or counterions) makes it difficult to translate studies of one system into predictions for another. While the important variable of cavity size is explored here, simulations on only one nanocavity type-rigid, spherical hydrophobic cavities-are presented. A great deal more investigation will be required to develop a unified understanding of the chemical dynamics in the diverse set of confinement frameworks.

\section{ACKNOWLEDGMENTS}

The author thanks Professor Brian B. Laird for useful comments and Dr. Damien Laage for stimulating discussions in the initial stages of this work. The computer facilities of the Kansas Center for Advanced Scientific Computing were used to carry out some of the calculations.

${ }^{1}$ C. J. Brinker and G. W. Scherer, Sol-Gel Science: The Physics and Chemistry of Sol-Gel Processing (Academic, New York, 1990).

${ }^{2}$ See, e.g., J. Rebek, Jr., Acc. Chem. Res. 32, 278 (1999); L. R. MacGillivray and J. L. Atwood, Adv. Supramolec. Chem. 6, 157 (2000).

${ }^{3}$ See, e.g., J. H. Fendler, J. Phys. Chem. 84, 1485 (1980); Structure and Reactivity in Reverse Micelles, edited by M. P. Pileni (Elsevier, New York, 1989).

${ }^{4}$ A recent example is L.-Q. Gu, S. Cheley, and H. Bayley, Science 291, 636 (2001).

${ }^{5}$ R. A. Marcus, Annu. Rev. Phys. Chem. 15, 155 (1964); M. D. Newton and N. Sutin, ibid. 35, 437 (1984)

${ }^{6}$ See, e.g., R. A. Marcus, Proc. Electrochem. Soc. 80, 1 (1980); A. Warshel, Computer Modelling of Chemical Reactions in Proteins and Solution (Wiley, New York, 1991); J. Juanós i Timoneda and J. T. Hynes, J. Phys. Chem. 95, 10431 (1991); P. L. Geissler, C. Dellago, D. Chandler, J. Hutter, and M. Parrinello, Science 291, 2121 (2001).

${ }^{7}$ J. Zhang and J. Jonas, J. Phys. Chem. 97, 8812 (1993); J.-P. Korb, S. Xu, and J. Jonas, J. Chem. Phys. 98, 2411 (1993); J.-P. Korb, L. Malier, F. Cros, S. Xu, and J. Jonas, Phys. Rev. Lett. 77, 2312 (1996); J.-P. Korb, S. Xu, F. Cros, L. Malier, and J. Jonas, J. Chem. Phys. 107, 4044 (1997).

${ }^{8}$ B. J. Loughnane, A. Scodinu, and J. T. Fourkas, J. Phys. Chem. B 103, 6061 (1999); B. J. Loughnane, A. Scodinu, R. A. Farrer, J. T. Fourkas, and U. Mohanty, J. Chem. Phys. 111, 2686 (1999); B. J. Loughnane, R. A. Farrer, A. Scodinu, T. Reilly, and J. T. Fourkas, J. Phys. Chem. B 104, 5421 (2000).

${ }^{9}$ C. Streck, Y. B. Mel'nichenko, and R. Richert, Phys. Rev. B 53, 5341 (1996); R. Richert, ibid. 54, 15762 (1996).

${ }^{10}$ K. Das, N. Sarkar, S. Das, A. Datta, and K. Bhattacharyya, Chem. Phys. Lett. 249, 323 (1996).

${ }^{11}$ N. Sarkar, K. Das, A. Datta, S. Das, and K. Bhattacharyya, J. Phys. Chem. 100, 10523 (1996); N. Sarkar, A. Datta, S. Das, and K. Bhattacharyya, ibid. 100, 15483 (1996); A. Datta, D. Mandal, S. K. Pal, and K. Bhattacharyya, J. Phys. Chem. B 101, 10221 (1997).

${ }^{12}$ S. K. Pal, D. Sukul, D. Mandal, S. Sen, and K. Bhattacharyya, J. Phys. Chem. B 104, 2613 (2000).

${ }^{13}$ A. Datta, S. K. Pal, D. Mandal, and K. Bhattacharyya, J. Phys. Chem. B 102, 6114 (1998).

${ }^{14}$ R. E. Riter, D. M. Willard, and N. E. Levinger, J. Phys. Chem. B 102, 2705 (1998).

${ }^{15}$ D. Pant, R. E. Riter, and N. E. Levinger, J. Chem. Phys. 109, 9995 (1998).

${ }^{16}$ R. E. Riter, E. P. Undiks, J. R. Kimmel, and N. E. Levinger, J. Phys. Chem. B 102, 7931 (1998).

${ }^{17}$ D. M. Willard, R. E. Ritter, and N. E. Levinger, J. Am. Chem. Soc. 120, 4151 (1998); D. M. Willard and N. E. Levinger, J. Phys. Chem. B 104, 11075 (2000).

${ }^{18}$ D. Pant and N. E. Levinger, Langmuir 16, 10123 (2000).

${ }^{19}$ R. E. Riter, E. P. Undiks, and N. E. Levinger, J. Am. Chem. Soc. 120, 6062 (1998)

${ }^{20}$ R. E. Riter, J. R. Kimmel, E. P. Undiks, and N. E. Levinger, J. Phys. Chem. B 101, 8292 (1997).

${ }^{21}$ J. Zhang and F. V. Bright, J. Phys. Chem. 95, 7900 (1991).

${ }^{22}$ C. H. Cho, M. Chung, J. Lee, T. Nguyen, S. Singh, M. Vedamuthu, S. Yao, 
J.-B. Zhu, and G. W. Robinson, J. Phys. Chem. 99, 7806 (1995).

${ }^{23}$ K. Hara, H. Kuwabara, and O. Kajimoto, J. Phys. Chem. A 105, 7174 (2001).

${ }^{24}$ P. Hazra and N. Sarkar, Chem. Phys. Lett. 342, 303 (2001).

${ }^{25}$ B. L. Bales, R. Ranganathan, and P. C. Griffiths, J. Phys. Chem. B 105, 7465 (2001).

${ }^{26}$ R. M. Dickson, D. J. Norris, Y.-L. Tzeng, and W. E. Moerner, Science 274, 966 (1996).

${ }^{27}$ H. Wang, A. M. Bardo, M. M. Collinson, and D. A. Higgins, J. Phys. Chem. B 102, 7231 (1998); E. Mei, A. M. Bardo, M. M. Collinson, and D. A. Higgins, ibid. 104, 9973 (2000).

${ }^{28}$ S. Das, A. Datta, and K. Bhattacharyya, J. Phys. Chem. A 101, 3299 (1997); D. Mandal, S. K. Pal, and K. Bhattacharyya, ibid. 102, 9710 (1998); S. K. Pal, D. Mandal, and K. Bhattacharyya, J. Phys. Chem. B 102, 11017 (1998)

${ }^{29}$ B. Valeur and E. Bardez, in Structure and Reactivity in Reverse Micelles, edited by M. P. Pileni (Elsevier, New York, 1989), pp. 103-122.

${ }^{30}$ Molecular Dynamics in Restricted Geometries, edited by J. Klafter and J. M. Drake (Wiley, New York, 1989); M. Schoen, Computer Simulation of Condensed Phases in Complex Geometries (Springer-Verlag, New York, 1993).

${ }^{31}$ N. Nandi and B. Bagchi, J. Phys. Chem. 100, 13914 (1996); K. Bhattacharyya and B. Bagchi, J. Phys. Chem. A 104, 10603 (2000).
${ }^{32}$ D. Brown and J. H. R. Clarke, J. Phys. Chem. 92, 2881 (1988).

${ }^{33}$ P. Linse, J. Chem. Phys. 90, 4992 (1989); P. Linse and B. Halle, Mol. Phys. 67, 537 (1989).

${ }^{34}$ J. Faeder and B. M. Ladanyi, J. Phys. Chem. B 104, 1033 (2000).

${ }^{35}$ J. Faeder and B. M. Ladanyi, J. Phys. Chem. B 105, 11148 (2001).

${ }^{36}$ S. Senapati and A. Chandra, J. Chem. Phys. 111, 1223 (1999).

${ }^{37}$ S. Senapati and A. Chandra, J. Phys. Chem. B 105, 5106 (2001).

${ }^{38}$ C. H. Turner, J. K. Brennan, J. K. Johnson, and K. E. Gubbins, J. Chem. Phys. 116, 2138 (2002).

${ }^{39}$ S. Vajda, R. Jimenez, S. J. Rosenthal, V. Fidler, G. R. Fleming, and E. W. Castner, Jr., J. Chem. Soc., Faraday Trans. 91, 867 (1995).

${ }^{40}$ E. A. Carter and J. T. Hynes, J. Phys. Chem. 93, 2184 (1989); J. Chem. Phys. 94, 5961 (1991).

${ }^{41}$ F. F. M. Freitas, F. M. S. S. Fernandes, and B. J. C. Cabral, J. Phys. Chem. 99, 5180 (1995).

${ }^{42}$ W. L. Jorgensen and J. M. Briggs, Mol. Phys. 63, 547 (1988).

${ }^{43}$ See, e.g., D. A. McQuarrie, Statistical Mechanics (Harper Collins, New York, 1976).

${ }^{44}$ M. P. Allen and D. J. Tildesley, Computer Simulation of Liquids (Oxford University Press, New York, 1987).

${ }^{45}$ D. Frenkel and B. Smit, Understanding Molecular Simulation, from Algorithms to Applications (Academic, New York, 1996). 